\title{
Cancer Patient-Reported Experience Measures (PREMs) Regarding the Policies Implemented to Contain the Spread of Sars-CoV-2 and Vaccination Campaign at Veneto Institute of Oncology
}

\author{
Mario Caccese $\mathbb{D}^{1}$, Silvia Imbevaro ${ }^{2}$, Alessandra Feltrin ${ }^{3}$, Daniela Costardi ${ }^{4}, N_{a d i a}$ Giordano ${ }^{5}$, \\ Maristella Maran', Rosalba Martino', Ketti Ottolitri ${ }^{6}$, Malihe Shams ${ }^{3}$, Federica Vascon ${ }^{7}$, Anna Roma ${ }^{5}$, \\ Antonella Galiano', Marco Maruzzo', Dario Marino', Giuseppe Lombardi', Sara Lonardi (iD), \\ Antonella Brunello', Vittorina Zagonel' \\ 'Department of Oncology, Oncology I, Veneto Institute of Oncology IOV - IRCCS, Padua, Italy; ${ }^{2}$ General Directorate, Accreditation and \\ Acknowledgment Unit, Veneto Institute of Oncolog IOV - IRCCS, Padua, Italy; ${ }^{3}$ Health Department, Psycho-Oncology Unit, Veneto Institute of \\ Oncology IOV - IRCCS, Padua, Italy; ${ }^{4}$ Scientific Directorate, Patient Education and Empowerment, Veneto Institute of Oncology IOV - IRCCS, Padua, \\ Italy; ${ }^{5}$ Department of Oncology, Oncology 3, Veneto Institute of Oncology IOV - IRCCS, Padua, Italy; ${ }^{6}$ Health Department, Clinical Risk Office, \\ Veneto Institute of Oncology IOV - IRCCS, Padua, Italy; ${ }^{7}$ Department of Imaging and Health Physics, Radiotherapy Unit, Veneto Institute of Oncology \\ IOV - IRCCS, Padua, Italy
}

Correspondence: Mario Caccese, Department of Oncology, Oncology I, Veneto Institute of Oncology IOV - IRCCS, Via Gattamelata, 64, Padua, 35।28, Italy, Email mario.caccese@iov.Veneto.it

Purpose: The SARS-CoV-2 spread has impacted Healthcare systems. COVID-19 pandemic has had consequences for patients with cancer, being associated with delays in diagnosis, in treatment And follow-up care, increase in overall infection rates and higher mortality. A survey on COVID-19 and a vaccination-questionnaire were developed at different times of the outbreak, to evaluate cancer patient-reported experience measures (PREMs) on the policies implemented to reduce the infection from SARS-CoV-2 and on the timing and methods of COVID-19 vaccination.

Patients and Methods: The survey was distributed to all patients accessing the Institute during the "first-wave" Of the pandemic, evaluating patients' concerns about the pandemic, the pandemics' consequences on their cancer care, and their perception Of the measures adopted to limit the infection spread. The vaccination-questionnaire was proposed to $10 \%$ of the first 5297 cancer patients vaccinated with two doses of the Pfizer-BioNTechCOVID-19 vaccine. This questionnaire aimed at assessing the degree Of satisfaction with the Institutional vaccination campaign and vaccination-related adverse events.

Results: From May 18th 2020 to June 15th 2020 the survey was completed by 3238 patients. Most of the responders expressed concern on the pandemic yet acknowledging their oncological disease as a priority. Measures implemented were appreciated by patients. Telemedicine was positively evaluated and the absence of the caregiver during the visit did not determine discomfort for two thirds of patients. From March 6th 2021 to May 8th 2021 the vaccination-questionnaire was completed by 357 patients. The $98.8 \%$ were satisfied with the vaccination campaign . No serious vaccination-correlated adverse events were reported. No patient had to delay/discontinue chemotherapy due to vaccination.

Conclusion: PREMs during COVID-19 pandemic and related vaccination can provide important information to help reorganization of the health care systems for cancer care. Patients' feedback on the organizational changes implemented in the emergency period are essential for healthcare improvement and to help informed choices that are consistent with patients' needs.

Keywords: patients' perspective, PREMs, COVID-19, survey, SARS-CoV-2, cancer patients

\section{Introduction}

Cancer remains a leading cause of mortality, especially in high-income countries, with about 10 million cancer deaths globally. ${ }^{1}$ The coronavirus disease 2019 (COVID-19) global pandemic, caused by the Severe Acute Respiratory 
Syndrome Coronavirus 2 (SARS-CoV-2) has substantially disrupted cancer care delivery. During the first COVID-19 outbreak, declared a pandemic by the WHO on March 11th 2020, Italy was the first western Country to be affected, with northern Italy being highly struck from February to April 2020. On March 8th, 2020, the Italian government issued a first national decree which established the isolation of areas with the greatest number of cases ("red-zones") including Padua and other cities in the Veneto Region, preceding a whole Country lockdown which was declared on March 9th, 2020. Drastic measures at a global healthcare level have been undertaken, such as increasing the intensive care unit capacity, postponing non-urgent visits, and implementing tele-health, along with extensive restrictions of the public life. In this scenario, cancer care in Italy has been highly preserved, with specific recommendations issued on March 10th, 2020 requiring local healthcare authorities to identify and apply specific protocols in order to reduce risk for patients, and to allow them to receive proper and timely treatment, as well as for physicians, recommending the use of appropriate personal protective equipment; eventually, to identify specific strategies to grant the diagnostic-therapeutic path for patients who accidentally came in contact with subjects infected with SARS-CoV-2. Given the vulnerability of oncological patients due both to cancer itself and to treatment effects, which cannot be usually postponed in order to preserve its effectiveness, ${ }^{2}$ patients with cancer were initially considered at high risk for Sars-CoV-2 infection, ${ }^{3-5}$ though available evidence to date suggests that incidence rates of COVID-19 among cancer patients receiving active treatment are still fairly low even if higher than the general population, ranging between 1 and $4 \% .{ }^{6,7}$ Veneto Institute of Oncology in Padua is a high-volume Comprehensive Cancer Center in the Veneto region, being the hub for the Veneto Oncological Network, with referrals of many patients throughout the Region. Considering the above-mentioned assumptions, the priority of Veneto Institute of Oncology was to provide continuous care during the COVID-19 outbreak, maintaining active take in charge of patients along with the adoption of institutional policies aiming at reducing infections, ensuring sufficient resources, and providing safety for patients and healthcare staff, in order to deliver optimal cancer treatment.

These measures included medical and nursing triage for hospital access, restrictions for accompanying family members/caregivers, use of personal protective equipment for physicians, nurses, healthcare professionals and patients, sanitization of environments, ensuring proper physical distancing and performing swabs and isolation in case of suspected SARS-CoV-2 infection. These measures have been unchanged since the beginning of the health emergency and have not changed during the study period regardless of the trend of SARS-CoV-2 infections in Italy and in the Veneto region. In addition to these measures, the Institute has implemented home-delivery of oral drugs after telephone contact with patients to limit unnecessary access in hospital. Such measures were all communicated in advance and in an exhaustive manner to all patients and caregivers (individually upon entering the institution and through written communications accessible to all patients and caregivers) to make them aware of the needs that the health emergency imposed on all those who entered the Institute and to ensure the same level of awareness in all users. On December 21st 2020 effective COVID-19 vaccines became available. ${ }^{8,9}$ After the vaccination of healthcare professionals, priority for vaccination was given to frail subjects, including cancer patients, as suggested by scientific societies at international [American Society of Clinical Oncology (ASCO), ${ }^{10}$ European Society for Medical Oncology (ESMO) ${ }^{11}$ ] and national level. ${ }^{12}$ A massive anti-COVID-19 vaccination campaign was implemented by the Veneto Institute of Oncology to ensure that cancer patients were quickly and safely vaccinated.

In this pandemic scenario, which has put patients and healthcare professionals in an unexpected and unprecedented condition with the difficulty of combining a risk-mitigation approach, the safe continuation of oncological treatment as well as the uncertainties of patients regarding their condition, the perception and experiences of patients is fundamental to ensure a high-level quality of care.

These drastic changes in routine care practice undoubtedly have a strong impact on patients' experience, particularly in cancer patients, and assessing the experiences and level of satisfaction with these measures using Patient-Reported Experience Measures (PREMs), can serve as an excellent indicator of patients' perception of the quality of healthcare service $^{13}$ and can be an objective measure of integrated care that has the person as its fulcrum. ${ }^{14}$

\section{Materials and Methods}

An internal survey was developed at Veneto Institute of Oncology - Irccs to assess the impact of the COVID-19 pandemic on patients' perceptions about the undertaken risk-mitigation measures and their concerns about their own 
cancer care. The survey consisted of a 32-item questionnaire which was distributed to all patients who had at least one visit at Veneto Institute of Oncology - IRCCS. Beyond a first set of questions regarding demographic data, other two sets of questions investigated patients' concerns about the pandemic and its consequences on their cancer diagnosis and treatment, and their perception of the risk-mitigation measures adopted by the Institute. In detail, the questionnaire contained 3 socio-demographic questions, 8 Likert scale and 3 open-ended questions (full questionnaire in Supplementary Material). A further questionnaire regarding the degree of satisfaction with the COVID-19 vaccination campaign was developed to be administered to patients vaccinated at the institute, consisting of a section regarding demographic data, 6 questions on the degree of satisfaction regarding the organization of the vaccination campaign and the last 2 questions regarding the onset of any vaccine-related adverse events related. Both questionnaires were defined with the support of the psycho-oncological specialists and the quality Department of the Institute. Patients were asked to anonymously answer to the questions, and participation was voluntary. Regarding the general survey, patients were divided into 3 categories: patients referred for a first visit, patients on treatment, and patients in follow-up care. With regard to the vaccination questionnaire, participation was addressed either to patients in active treatment or to patients in follow-up care. All patients entering Veneto Institute of Oncology sign an informed consent to the processing of personal data and their health information for scientific research purposes in the medical, biomedical and epidemiological fields.

Descriptive statistics of the data as well as comparisons between groups were performed using " $\mathrm{R}$ " statistics software (version 3.2.3). The Pearson's chi square test was used to test for differences between specific groups. P-value $<0.05$ was considered statistically significant.

The study was approved by the Veneto Institute of Oncology Ethics Committee on May 7th, $2020\left(\mathrm{n}^{\circ}\right.$ 8494) and complied the Declaration of Helsinki.

\section{Results}

\section{General Survey}

From May 18th 2020 to June 15th 2020, out of 4.494 distributed surveys, a total of 3238 was returned (72\% - Figure 1A). Patients' characteristics are reported in Table 1.

\section{Concerns Regarding COVID-I9 Pandemic and Evaluation of the Contagion Containment Measures}

Degree of concern for the COVID-19 health emergency was assessed in the whole patient population and the possible answers were "little", "enough" or "a lot", with 16\% of patients reporting "little" concern, while 49\% and 35\% reported "enough" and "a lot" of concern respectively. The greatest degree of concern emerged in the age groups 50-65 and $>65$ years compared to younger patients $(p=0.002)$, in female patients $(p<0.0001)$ and in patients on treatment $(p>0.0001)$ (Table 2). Almost all participating patients considered the measures adopted by the Institute to reduce the risk of infection from SARS-CoV-2 as good or excellent, in particular, $92 \%$ and $94 \%$ of patients rated as good/excellent the medical and nursing triage for admission to hospital and the use of personal protective equipment for physicians, nurses, healthcare professionals and patients respectively; $90 \%$ of patients particularly appreciated the sanitizing procedures of the hospital rooms and $87 \%$ likewise considered the policies adopted to guarantee the proper physical distancing. Swabbing and isolation in case of suspected SARS-CoV-2 infection was rated good/excellent by $92 \%$ of patients of responding patients. Three quarters of the responding patients $(75 \%)$ stated they felt more protected thanks to the measures implemented by the Institute; $41 \%$ of the patients said that such measures had improved the quality of both medical and nursing care with a further $44 \%$ of patients who did not report any change in the quality of care (Figure 2). About two thirds of responding patients (74\%) agreed that some of the risk-mitigation measures should be maintained after the health emergency ends, in particular the use of personal protection, social distancing and regulated entry into the hospital. With regard to which one between cancer and COVID-19 caused the greatest concern, two-thirds (67\%) of patients reported more concern about their cancer diagnosis, in particular, male patients $(\mathrm{p}<0.0001)$ and those entering the Institute for the first time $(\mathrm{p}<$ $0.0001)$, whereas older patients $(>65$ years) reported greater concern for the infectious disease $(\mathrm{p}<0.0001)$. 


\section{A}

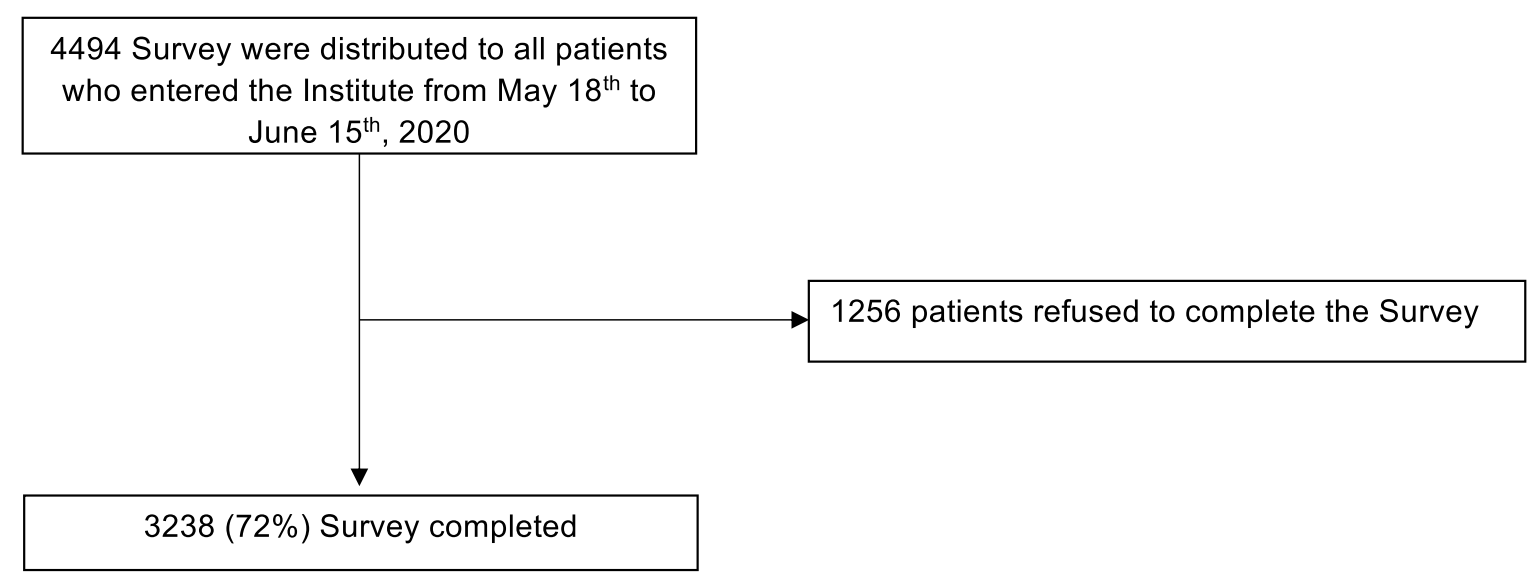

B

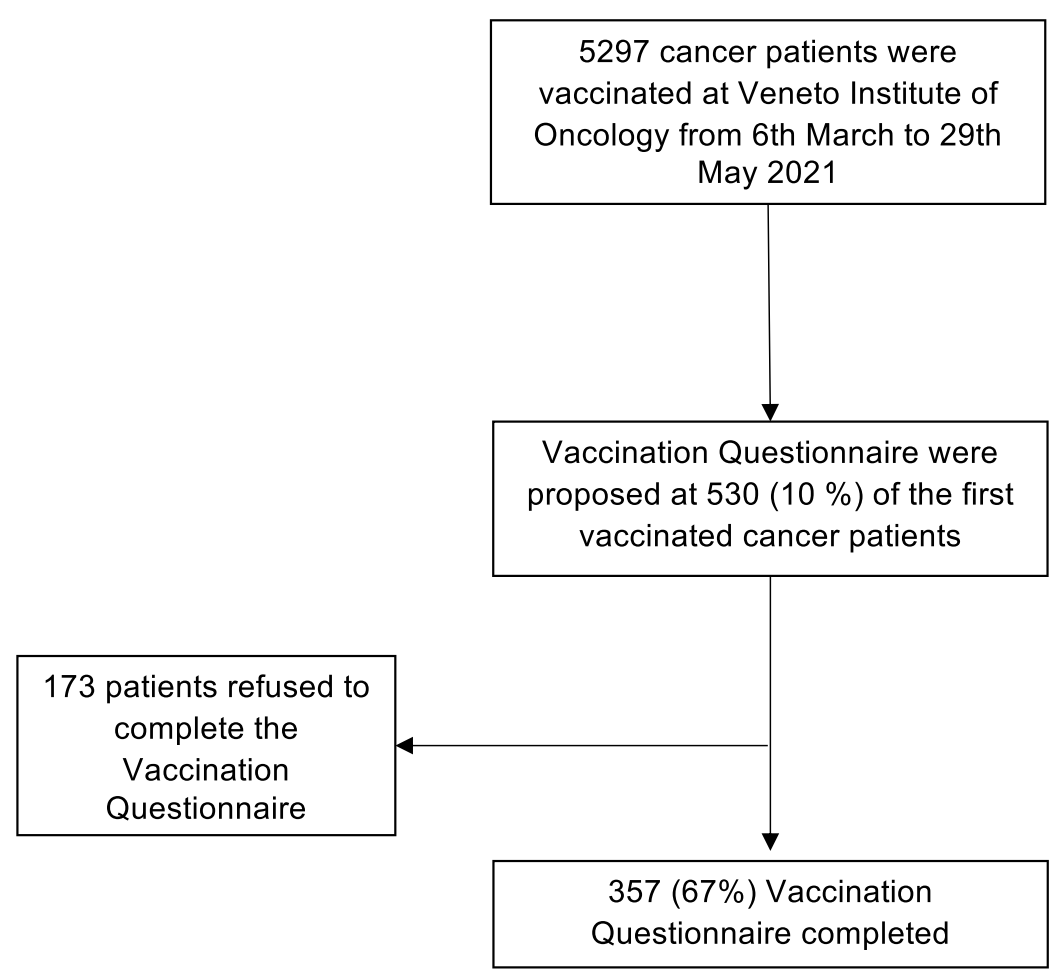

Figure I (A) Flow-chart of COVIDI9 survey. (B) Flow-chart of vaccination questionnaire.

\section{Telemedicine}

In the evaluated time-period, $29 \%$ of the patients stated that they had received more frequent telephone or email contacts from medical staff and, of these, more than $90 \%$ considered them particularly useful. Tele-health was implemented during the first outbreak as part of the risk mitigation policies to limit patients' access to hospital. Patients who received more contacts (telephone or email) were those under treatment compared to patients undergoing follow-up or to newly diagnosed patients with first access to the Institute $(\mathrm{p}<0.0001)$ and patients aged $>65$ years than younger ones $(\mathrm{p}=0.03)$. Overall, $63 \%$ of patients who had telemedicine contacts judged it to be comparable to a "face-to-face" visit regardless of age, gender, and treatment setting; only $12 \%$ of responders reported a negative evaluation of telemedicine (Figure 2), and the limitations of this approach that have emerged more frequently in patients' comments regard the need of direct contact with the medical staff for reassurance, as well as possible problems of telephone/internet accessibility. 
Table I Survey Patients' Characteristics

\begin{tabular}{|l|l|}
\hline Patients' Characteristics & N (\%) \\
\hline All Patients & $3.238(100)$ \\
Gender & $1.212(37.4)$ \\
$\quad$ Male & $1.951(60.3)$ \\
Female & $75(2.3)$ \\
Unknown (missing answer) & 60 \\
Median Age (years) & $16-98$ \\
Age (years), range & \\
Age & $698(21.6)$ \\
< 50 years & $1.187(36.7)$ \\
$50-65$ years & $1.114(34.4)$ \\
>65 years & $239(7.3)$ \\
Unknown (missing answer) & \\
Treatment setting & $1.701(52.5)$ \\
Undergoing treatment & $1.213(37.5)$ \\
Follow-up & $248(7.7)$ \\
New patient & $76(2.3)$ \\
Unknown (missing answer) &
\end{tabular}

Table 2 Level of Concern About COVID-19 Pandemic According to Age, Gender and Treatment Setting

\begin{tabular}{|c|c|c|c|c|c|c|c|c|}
\hline \multirow{2}{*}{$\begin{array}{l}\text { Level of Concern About the } \\
\text { COVID-19 Pandemic }\end{array}$} & \multicolumn{3}{|l|}{ Age } & \multicolumn{2}{|l|}{ Gender } & \multicolumn{2}{|c|}{ Treatment Setting } & \multirow[b]{2}{*}{ Follow-Up } \\
\hline & $\begin{array}{l}\leq \mathbf{5 0} \\
\text { Years }\end{array}$ & $\begin{array}{l}50-65 \\
\text { Years }\end{array}$ & $\geq 65$ Years & Female & Male & $\begin{array}{l}\text { First } \\
\text { Access }\end{array}$ & $\begin{array}{l}\text { Under } \\
\text { Treatment }\end{array}$ & \\
\hline Little & $18 \%$ & $15 \%$ & $15 \%$ & $14 \%$ & $20 \%$ & $18 \%$ & $15 \%$ & $17 \%$ \\
\hline Enough & $51 \%$ & $49 \%$ & $45 \%$ & $47 \%$ & $48 \%$ & $49 \%$ & $44 \%$ & $51 \%$ \\
\hline A lot & $28 \%$ & $34 \%$ & $36 \%$ & $36 \%$ & $28 \%$ & $28 \%$ & $38 \%$ & $28 \%$ \\
\hline Unknown & $3 \%$ & $3 \%$ & $4 \%$ & $3 \%$ & $4 \%$ & $5 \%$ & $3 \%$ & $4 \%$ \\
\hline Total & $100 \%$ & $100 \%$ & $100 \%$ & $100 \%$ & $100 \%$ & $100 \%$ & $100 \%$ & $100 \%$ \\
\hline & & & $\mathrm{p}$-value $=0.002$ & \multicolumn{2}{|c|}{$\mathrm{p}$-value $<0.000 \mathrm{I}$} & & & $\mathrm{p}$-value $<0.000 \mathrm{I}$ \\
\hline
\end{tabular}

\section{Family/Caregiver Presence and Visitor Restrictions}

Hospital access for family members/caregivers were not permitted to limit the number of subjects inside the Institute as part of the contagion-limiting policy. Patients with limited mobility or autonomy could count on healthcare and voluntary staff for assistance. Overall, 71\% of patients reported not being distressed with such measure, with those experiencing more discomfort being patients older than 65 years (vs younger ones, $p=0.003$ ), female $(p=0.006)$, patients who reported a high rate of concern for the COVID-19 health emergency $(\mathrm{p}<0.0001)$ as well as patients under treatment or newly diagnosed at their first access to the Institute compared to those in follow-up care $(\mathrm{p}<0.0001)$. Patients' discomfort due to the absence of a caregiver mainly regarded the loss of emotional support, feelings of anxiety and solitude as well as the absence of company during waiting times.

\section{Vaccine Questionnaire}

The questionnaires regarding the vaccination campaign were distributed from April 26th 2021 to May 14th 2021 to a cohort of 530 patients $(10 \%)$ of the 5297 cancer patients who received the vaccine in the Institute from March to May 2021 (two 30 ug doses of the BNT162b2 Pfizer-BioNTech Covid vaccine), and 357 questionnaires were returned (67\%) (Figure 1B). Patients' characteristics are reported in Table 3. The first set of questions assessed the degree of 

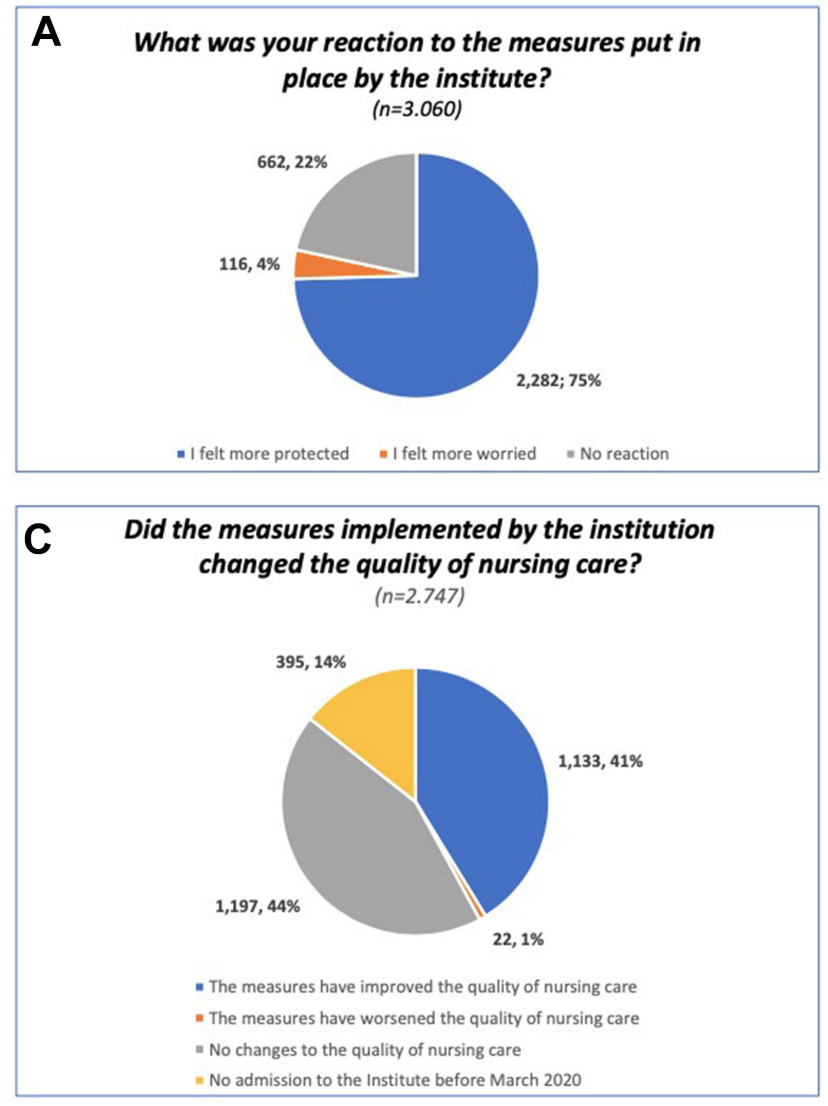
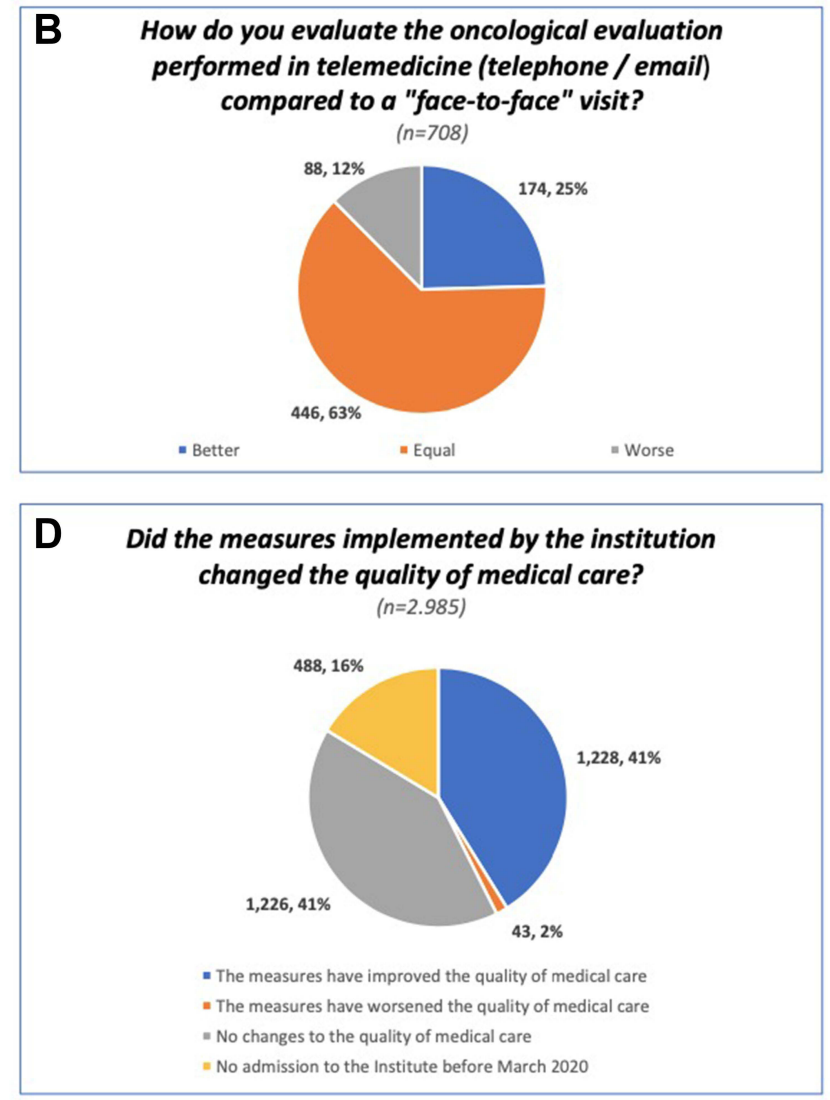

Figure 2 Reaction to the measures taken by the institution (A), telemedicine (B) and assessment of the quality of nursing (C)/medical (D) care.

satisfaction with the vaccination proposal by oncologists and the clarity, availability, and completeness of information regarding the vaccine (method of administration, purpose and potential adverse events): $97.1 \%$ and $95.5 \%$ of the respondents were very satisfied/satisfied with the proposal by oncologists and with the clarity and completeness of the information provided respectively. Furthermore, $86.5 \%$ of responding patients felt very satisfied/satisfied with the clarity and efficacy of the information received regarding the home management of adverse events related to the administration of the vaccine. With regard to side effects, $33 \%$ of responders stated they experienced minor side effects after the

Table 3 Vaccination Questionnaire Patients' Characteristics

\begin{tabular}{|l|l|}
\hline Patients' Characteristics & N (\%) \\
\hline All Patients & $357(100)$ \\
Gender & \\
Male & $140(39.2)$ \\
Female & $212(59.3)$ \\
Unknown (missing answer) & $5(1.5)$ \\
Median Age (years) & 68.5 \\
Age (years), range & $20-90$ \\
Treatment setting & \\
Undergoing treatment & $279(78)$ \\
Follow-up & $30(8.4)$ \\
Unknown (missing answer) & $48(13.6)$ \\
\hline
\end{tabular}


administration of the vaccine, in particular $7 \%$ only after the first dose, $49 \%$ only after the second while $44 \%$ after both doses. No serious vaccination-correlated adverse events were detected.

\section{Discussion}

We herein report the results of Veneto Institute of Oncology - Irccs survey about impact of the Covid-19 pandemic on cancer patients. Veneto Institute of Oncology has been engaged since the beginning of the pandemic in assessing epidemiology and clinical course of COVID-19 in cancer patients, with a first report by Guarneri et al ${ }^{15}$ describing the characteristics of patients with cancer and SARS-CoV-2 infection within the Veneto Oncological Network. Out of 170 patients, hospitalization was necessary for $77 \%$, with $6 \%$ requiring intensive care Unit admission; at the time of the analysis $33 \%$ of the patients had died and $17 \%$ of these from causes directly related to the infection. At the ASCO 2021 annual meeting, comparative data between the first and second wave from COVID 19 in terms of epidemiology and clinical course were presented, showing important differences in patient and tumor-type characteristics and confirming the role of reduction of in-hospital contact as a source of infection, which reflects the efforts put in place to protect this vulnerable population. ${ }^{16}$ Several papers have then addressed the management of older patients with cancer and the risk of infection from SARS-CoV-2, ${ }^{17}$ the definition of the risk-benefit ratio in the treatment of patients with cancer beyond the second line of treatment during the COVID19 pandemic, ${ }^{18}$ the benefit and safety of high-dose cancer treatments and autologous stem cell transplantation, ${ }^{19}$ the implications of pandemics and infection in patients with brain tumors ${ }^{20}$ and lung cancers. ${ }^{21}$ Ensuring safety and measuring the quality of care are mainstays of Oncology practice. Patient reported experience measures (PREMs) have been increasingly used in various health fields for assessing the quality of health care perceived by patients. ${ }^{14,22-25}$ The role of collecting PREMs during a totally unique event as the COVID-19 pandemic carries stimulating and innovative aspects. Previous studies have evaluated the perspective of cancer patients regarding the COVID-19 pandemic, cancer treatments and organizational challenges in oncological acitivity ${ }^{26-28}$ but, to our knowledge, this is one of the largest reports on patients' perceptions regarding the measures taken to limit the risk of SARS-CoV-2 infection along with their concern about their own cancer care, and the first to acknowledge patients' satisfaction on oncologists' initiated vaccination campaign. All the measures implemented by the Institute to reduce the risk of contagion and to guarantee a "COVID-free" cancer center have been maintained since the beginning of the health emergency and are still ongoing.

Participation to the survey was on a voluntary basis, yet $72 \%$ of patients returned the questionnaire, and such a high rate of adhesion denotes the importance of this issue to cancer patients, with an overall high prevalence of concerns about the risks of the COVID-19 pandemic, intense awareness of the need for restrictive measures to reduce the spread of SARS-CoV-2 infection, while keeping the concern for oncological disease as a priority.

Patients who participated in the survey were comforted and reassured by risk-mitigation policies put in place in the Institute, globally agreeing on the measures applied demonstrating strong resilience.

The restriction measures limiting access for caregivers, as expected, impacted physician-patient relationship, yet this turned out to cause discomfort for only a minority of patients, while the majority of patients did not report being affected by such a limitation which would seem to have favored a more direct and "exclusive" physician-patient relationship. Patients who suffered the most by the lack of a caregiver stressed the need for emotional support to cope with feelings of anxiety, loss, fear, and loneliness as well as the need for physical assistance or help in understanding and remembering medical indications. In particular, the absence of an accompanying caregiver was an issue particularly for older patients. Considering the high prevalence of cancer in older subjects and the greater risk of a worse outcome of SARS-CoV-2 infection in this population, a multidimensional assessment to guide decisions and create a safe environment is of fundamental importance for an optimal management. ${ }^{17}$ Median age of the respondents in our survey was 60 years, with $37 \%$ of patients being older than 65 years. Recent data point to a high level of concern of patients about the wide community spread of COVID-19, ${ }^{29}$ and this finding was confirmed by our survey, with older patients being the more affected. The same study found out that caregivers were primarily worried about patients dying alone, a possibility which is extremely concrete in case patients with high burden of disease getting infected. ${ }^{18}$ Our survey also investigated opinions on implementation of telemedicine in cancer care. Tele-health has been of great help during the pandemic, since it has enabled physicians to follow-up with patients, and at the same time limit their risk of exposure to potential 
infection. One of the greatest concerns with a rapid adoption of telemedicine is maintaining the safety and quality of care ${ }^{30}$ nonetheless data from this survey show that patient's perception of the quality of the services provided remained very high. Globally, most oncological centers have resorted to telemedicine for follow-up visits, ${ }^{31,32}$ yet only few data are available on patients' reception of these changes in their care. In particular, very little is known on whether telemedicine meets the demands of cancer patients. Our data show that telephone/email contacts increased by $29 \%$ in the analysis period and that it was rated very positively and, in many cases, $(63 \%)$ was even considered comparable to a "face-toface" visit both in terms of effectiveness and accessibility. Patients who negatively rated a telemedicine approach (12\%) underlined the limits of telephone/e-mail contact, judging a "face-to-face" visit to be more effective because it was more thorough and reassuring. The majority of patients stated that measures implemented during the pandemic should be maintained after the health emergency is over, in order to ensure greater safety for patients and healthcare professionals; however, this proposal would require a review of the organization of hospital cancer services as well as economic and human resources. With regard to telemedicine, evidence for its acceptance by older patients is scarce, with a recent systematic review in a non-oncological setting showing there were high levels of satisfaction across several assessed domains relating to telemedicine ${ }^{33}$ and some early data in the general cancer population pointing at a fair general acceptance of telemedicine, as well as of tele-health multidisciplinary geriatric oncology clinics. ${ }^{34}$ Tele-health has been shown to improve patient outcomes in non-oncological settings especially by lowering re-admission rates, which is of pivotal importance for older patients during the pandemic. ${ }^{35}$ These data are in line with those presented at recent ESMO congress in the Oncotelemed Trial, ${ }^{36}$ highlighting high patients' satisfaction for virtual visits, with, however, older patients having less knowledge of new technologies and preferring telephone contacts over videocalls and/or e-mails. Our data, on the other hand, did show different experience reported by older compared to younger subjects, and this finding paves to way to further implementation of tele-health for this population of patients at highest risk of infection. Given this background, telehealth services should be encouraged during the COVID-19 pandemic, in particular for older patients for delivering geriatric assessment, ${ }^{37}$ for management of treatment-related toxicity, follow-up care, as well as for pre-habilitation programs. ${ }^{38}$ Vaccination against SARS-CoV-2 is providing a shield and likely changing the natural history of the pandemic, therefore adherence to the vaccination campaign is of fundamental importance, especially for cancer patients who have an increased risk of complications from COVID-19. ${ }^{39}$ In our experience, patients who, adequately informed, refused vaccination was less than 5\%, way less than data in the literature, which report refusal rates over $10 \%{ }^{40,41}$ This might be explained by the campaign we held, that involved only professional figures of the Institute, with enrollment via proposal by treating oncologists, also testified by the high level of satisfaction shown by the patients themselves with the vaccination questionnaire.

With its limits, one of the major ones being not having data on type of cancer and stage of disease which might have helped to better contextualize some responses, this study shed some lights into Patient-Reported Experience Measures (PREMs) and feelings concerning the pandemic and antiCOVID-19 vaccines, along with providing an evidence base which comforts healthcare personnel in pursuing patients' best interests during the future healthcare challenges. The proposal of the general survey after the first wave of the COVID-19 pandemic could constitute another limitation of this study, since at that time Italy and the Veneto region were exiting the lockdown and the number of SARS-CoV-2 infections was significantly lower compared to the previous period; the administration of this questionnaire in the midst of a pandemic wave, such as the one we are experiencing in this period, would likely lead to different perceptions of cancer patients in the different phases of the pandemic. Moreover, this study does not take into consideration other implemented measures, such as prioritization of cancer treatment and home delivery of oral drugs since questionnaires were administered only to patients physically present at the Institute.

\section{Conclusions}

Our data on a general survey and vaccine questionnaire provide us with a realistic overview on cancer patients-reported experiences measures regarding the risk-reducing policies undertaken during the COVID-19 pandemic and the COVID19 vaccination campaign in a Comprehensive Cancer Center in Italy. Notwithstanding intrinsic limits of these data, they serve as a guide as to how we can best support our patients during the pandemic in relation to the expressed needs. Involving all stakeholders, among whom patients represent a fundamental voice, in the organizational re-planning of 
services can lead to a truly "personalized medicine", and this in turn may help mitigate the negative effects of the ongoing and future healthcare challenges, as well as helping healthcare providers in their daily activities and help informed choices that are consistent with patients' needs.

\section{Acknowledgments}

We thank all the patients who participated in the survey. These authors have to be considered as senior co-authors: Antonella Brunello and Vittorina Zagonel.

\section{Disclosure}

Dr Sara Lonardi reports grants and personal fees from Amgen, Merck Serono Roche, Lilly, Astra Zeneca, and BristolMyers Squibb, grants from Bayer, and personal fees from Incyte, Daiichi-Sankyo, Servier, MSD, Pierre-Fabre, and GSK, outside the submitted work. Dr Antonella Brunello reports personal fees and travel expenses from and advisory board for Pharmamar, and personal fees from Glaxo Smith Kline, outside the submitted work. The authors report no other potential conflicts of interest for this work.

\section{References}

1. Bray F, Ferlay J, Soerjomataram I, et al. Global cancer statistics 2018: GLOBOCAN estimates of incidence and mortality worldwide for 36 cancers in 185 countries. CA Cancer J Clin. 2018;68:394-424. doi:10.3322/caac.21492

2. Lewis MA. Between scylla and charybdis - oncologic decision making in the time of Covid-19. N Engl J Med. 2020;382:2285-2287. doi:10.1056/ NEJMp2006588

3. Tian J, Yuan X, Xiao J, et al. Clinical characteristics and risk factors associated with COVID-19 disease severity in patients with cancer in Wuhan, China: a multicentre, retrospective, cohort study. Lancet Oncol. 2020;21:893-903. doi:10.1016/S1470-2045(20)30309-0

4. Dai M, Liu D, Liu M, et al. Patients with cancer appear more vulnerable to SARS-CoV-2: a multicenter study during the COVID-19 outbreak. Cancer Discov. 2020;10:783-791. doi:10.1158/2159-8290.CD-20-0422

5. Liang W, Guan W, Chen R, et al. Cancer patients in SARS-CoV-2 infection: a nationwide analysis in China. Lancet Oncol. 2020;21:335-337. doi:10.1016/S1470-2045(20)30096-6

6. Rogado J, Obispo B, Pangua C, et al. Covid-19 transmission, outcome and associated risk factors in cancer patients at the first month of the pandemic in a Spanish hospital in Madrid. Clin Transl Oncol. 2020;22:2364-2368. doi:10.1007/s12094-020-02381-z

7. Bertuzzi AF, Marrari A, Gennaro N, et al. Low incidence of SARS-CoV-2 in patients with solid tumours on active treatment: an observational study at a tertiary cancer centre in Lombardy, Italy. Cancers (Basel). 2020;12:E2352. doi:10.3390/cancers12092352

8. European Medicines Agency.EMA recommends first COVID-19 vaccine for authorisation in the EU; 2020. Available from: https://www.ema. europa.eu/en/news/ema-recommends-first-covid-19-vaccine-authorisation-eu. Accessed January 25, 2022.

9. US Food \& Drug Administration. Comirnaty and Pfizer-BioNTech COVID-19 Vaccine; 2022.Available from: https://www.fda.gov/emergencypreparedness-and-response/coronavirus-disease-2019-covid-19/pfizer-biontech-covid-19-vaccine. Accessed January 25, 2022.

10. ASCO. COVID-19 vaccine \& patients with cancer; 2020. Available from: https://www.asco.org/asco-coronavirus-resources/covid-19-patient-careinformation/covid-19-vaccine-patients-cancer-information/covid-19-vaccine-patients-cancer. Accessed January 25, 2022.

11. European Society for Medical Oncology. ESMO call to action on COVID-19 vaccinations and patients with cancer: vaccinate. Monitor. Educate. Available from: https://www.esmo.org/policy/esmo-call-to-action-on-covid-19-vaccinations-and-patients-with-cancer-vaccinate-monitor-educate. Accessed January 25, 2022.

12. Silvestris N, Di Maio M, Russo A, et al. COVID-19 infection in cancer patients: what has been the contribution of Associazione Italiana Oncologia Medica (AIOM) to oncological care since the beginning of the first pandemic wave? ESMO Open. 2021;6:100100. doi:10.1016/j. esmoop.2021.100100

13. Koehler WF, Fottler MD, Swan JE. Physician-patient satisfaction: equity in the health services encounter. Med Care Rev. 1992;49:455-484. doi: $10.1177 / 002570879204900404$

14. Cinocca S, Rucci P, Randazzo C, et al. Validation of the Italian version of the patient reported experience measures for intermediate care services. PPA. 2017;11:1671-1676. doi:10.2147/PPA.S140041

15. Guarneri V, Bassan F, Zagonel V, et al. Epidemiology and clinical course of severe acute respiratory syndrome coronavirus 2 infection in cancer patients in the Veneto Oncology Network: the Rete Oncologica Veneta covID19 study. Eur J Cancer. 2021;147:120-127. doi:10.1016/j. ejca.2021.01.021

16. Guarneri V, Caccese M, Azzarello G, et al. Epidemiology and clinical course of SARS-CoV-2 infection in cancer patients in the Veneto Oncology Network during the first and second pandemic waves. JCO. 2021;39:6511. doi:10.1200/JCO.2021.39.15_suppl.6511

17. Brunello A, Galiano A, Finotto S, et al. Older cancer patients and COVID-19 outbreak: practical considerations and recommendations. Cancer Med. 2020;9:9193-9204. doi:10.1002/cam4.3517

18. Brunello A, Zagonel V. Caring for patients with advanced stage cancer at the time of COVID-19. Oncologist. 2020;25:e1131. doi:10.1634/ theoncologist.2020-0230

19. Marino D, Finotto S, Basso U, et al. To transplant or not to transplant during the SARS-CoV-2 pandemic? That is the question. Oncologist. 2021;26:e336-e337. doi:10.1002/onco.13563

20. Simonelli M, Franceschi E, Lombardi G. Neuro-oncology during the COVID-19 outbreak: a hopeful perspective at the end of the Italian crisis. Front Med (Lausanne). 2020;7:594610. doi:10.3389/fmed.2020.594610 
21. Pasello G, Menis J, Pilotto S, et al. How the COVID-19 pandemic impacted on integrated care pathways for lung cancer: the parallel experience of a COVID-spared and a COVID-dedicated center. Front Oncol. 2021;11:669786. doi:10.3389/fonc.2021.669786

22. Male L, Noble A, Atkinson J, Marson T. Measuring patient experience: a systematic review to evaluate psychometric properties of patient reported experience measures (PREMs) for emergency care service provision. Int J Qual Health Care. 2017;29:314-326. doi:10.1093/intqhe/mzx027

23. Brown A, Ford T, Deighton J, Wolpert M. Satisfaction in child and adolescent mental health services: translating users' feedback into measurement. Adm Policy Ment Health. 2014;41:434-446. doi:10.1007/s10488-012-0433-9

24. Bosworth A, Cox M, O'Brien A, et al. Development and validation of a Patient Reported Experience Measure (PREM) for patients with Rheumatoid Arthritis (RA) and other rheumatic conditions. Curr Rheumatol Rev. 2015;11:1-7. doi:10.2174/1573397111666150522093712

25. Lagha E, Noble A, Smith A, Denvir MA, Leslie SJ. Patient Reported Experience Measures (PREMs) in chronic heart failure. J R Coll Physicians Edinb. 2012;42:301-305. doi:10.4997/JRCPE.2012.404

26. Zuliani S, Zampiva I, Tregnago D, et al. Organisational challenges, volumes of oncological activity and patients' perception during the severe acute respiratory syndrome coronavirus 2 epidemic. Eur J Cancer. 2020;135:159-169. doi:10.1016/j.ejca.2020.05.029

27. de Joode K, Dumoulin DW, Engelen V, et al. Impact of the coronavirus disease 2019 pandemic on cancer treatment: the patients' perspective. Eur $J$ Cancer. 2020;136:132-139. doi:10.1016/j.ejca.2020.06.019

28. Mulvey TM, Jacobson JO. COVID-19 and cancer care: ensuring safety while transforming care delivery. JCO. 2020;38:3248-3251. doi:10.1200/ JCO.20.01474

29. Ng KYY, Zhou S, Tan SH, et al. Understanding the psychological impact of COVID-19 pandemic on patients with cancer, their caregivers, and health care workers in Singapore. JCO Global Oncol. 2020;1494-1509. doi:10.1200/GO.20.00374

30. Royce TJ, Sanoff HK, Rewari A. Telemedicine for cancer care in the time of COVID-19. JAMA Oncol. 2020;6:1698. doi:10.1001/ jamaoncol.2020.2684

31. Freyer FJ. Forced by the Pandemic, Health Care Plunges into the Digital Age. Boston Globe; 2020

32. Lonergan PE, Washington SL, Branagan L, et al. Rapid utilization of telehealth in a comprehensive cancer center as a response to COVID-19: cross-sectional analysis. J Med Internet Res. 2020;22:e19322. doi:10.2196/19322

33. Orlando JF, Beard M, Kumar S, Borsci S. Systematic review of patient and caregivers' satisfaction with telehealth videoconferencing as a mode of service delivery in managing patients' health. PLoS One. 2019;14:e0221848. doi:10.1371/journal.pone.0221848

34. Wall SA, Knauss B, Compston A, et al. Multidisciplinary telemedicine and the importance of being seen. J Geriatr Oncol. 2020;11:1349-1351. doi:10.1016/j.jgo.2020.05.006

35. O'Connor M, Asdornwised U, Dempsey M, et al. Using telehealth to reduce all-cause 30-day hospital readmissions among heart failure patients receiving skilled home health services. Appl Clin Inform. 2016;7:238-247. doi:10.4338/ACI-2015-11-SOA-0157

36. Ribera P, Climent C, Soriano S, et al. Patient preferences towards the application of telemedicine on cancer care during Coronavirus disease 2019 (COVID-19) pandemic. ONCOTELEMED STUDY. Ann Oncol. 2021;32:S1159-S1160.

37. DiGiovanni G, Mousaw K, Lloyd T, et al. Development of a telehealth geriatric assessment model in response to the COVID-19 pandemic. J Geriatr Oncol. 2020;11:761-763. doi:10.1016/j.jgo.2020.04.007

38. Sell NM, Silver JK, Rando S, et al. Prehabilitation telemedicine in neoadjuvant surgical oncology patients during the novel COVID-19 coronavirus pandemic. Ann Surg. 2020;272:e81-e83. doi:10.1097/SLA.0000000000004002

39. Kuderer NM, Choueiri TK, Shah DP, et al. Clinical impact of COVID-19 on patients with cancer (CCC19): a cohort study. Lancet. 2020;395:1907-1918. doi:10.1016/S0140-6736(20)31187-9

40. Di Noia V, Renna D, Barberi V, et al. The first report on coronavirus disease 2019 (COVID-19) vaccine refusal by patients with solid cancer in Italy: early data from a single-institute survey. Eur J Cancer. 2021;153:260-264. doi:10.1016/j.ejca.2021.05.006

41. Moujaess E, Zeid NB, Samaha R, et al. Perceptions of the COVID-19 vaccine among patients with cancer: a single-institution survey. Future Oncol. 2021;17:4071-4079. doi:10.2217/fon-2021-0265

\section{Publish your work in this journal}

Patient Preference and Adherence is an international, peer-reviewed, open access journal that focusing on the growing importance of patient preference and adherence throughout the therapeutic continuum. Patient satisfaction, acceptability, quality of life, compliance, persistence and their role in developing new therapeutic modalities and compounds to optimize clinical outcomes for existing disease states are major areas of interest for the journal. This journal has been accepted for indexing on PubMed Central. The manuscript management system is completely online and includes a very quick and fair peer-review system, which is all easy to use. Visit http://www.dovepress.com/testimonials.php to read real quotes from published authors.

Submit your manuscript here: https://www.dovepress.com/patient-preference-and-adherence-journal 